\title{
The Capacity Region of a Class of Deterministic $Z$ Channels
}

\author{
Viveck R. Cadambe, Syed A. Jafar \\ Electrial Engineering and Computer Science \\ University of California, Irvine \\ Email: \{vcadambe, syed\}@uci.edu
}

\author{
Sriram Vishwanath \\ Department of Electrical and Computer Engineering \\ University of Texas, Austin \\ Email: sriram@ece.utexas.edu
}

\begin{abstract}
We characterize the capacity region of a class of the deterministic $Z$ channels. We show that, interestingly, Han-Kobayashi type rate-splitting is not required in the optimal achievable scheme for the class of channels considered.
\end{abstract}

\section{INTRODUCTION}

The capacity regions of multi-user scenarios which model communication networks are among the most important open problems in multi-user information theory. Specifically, the capacity regions of the broadcast, relay and interference channels are unknown in general. However, progress has been made in their capacity characterization when the outputs are deterministic functions of the inputs. The capacity region of the deterministic broadcast channel is known, even when there is a common message [1]. The capacity region of a class of deterministic interference channels has been characterized in [2], with the achievable strategy using the Han-Kobayashi scheme. The study of deterministic channels is receiving increased attention, of late, because of recently established connections between the capacity regions of certain deterministic channels and their Gaussian counterparts. In [3], the capacity region of the Gaussian interference channel has been approximated to within one bit, using insights obtained from the deterministic interference channels studied in [2], [4]. Deterministic channels have also helped approximate capacity regions of certain wireless relay networks [5][7], the fading broadcast channel [9], the many-to-one and one-to-many interference networks [8], and the fullyconnected $K$-user interference networks [10], [11]. Motivated by the connections between deterministic channels and their Gaussian counterparts, we study a class of deterministic $Z$ channels [12] in this paper. The class of channels considered in this paper is similar to the class of deterministic interference channels studied in [2].

This work was supported by NSF under Grant CCF-0830809 and by ONR YIP under Grant N00014-08-1-0872
The deterministic $Z$ channel (see Figure 1) consists of two distributed transmitters and two distributed receivers. The output of the first receiver is a function of the inputs at both transmitters. The output at the second receiver only depends on the input at the second transmitter, but is independent of the input at the first transmitter. There are three independent messages in the system. There is a message from the first transmitter to the first receiver. There are two messages at the second transmitter - one message corresponding to each receiver. Achievability and outerbound arguments have been provided in earlier works for the discrete memoryless $Z$ channels in [12], [13], and for the Gaussian $Z$ channel in [14].

The main result of this work is a single-letter characterization of the capacity region of a class of deterministic $Z$ channels. The outer bound follows from adapting bounds known for the Gaussian $Z$ channel (See [15]) to the deterministic setting. The achievable scheme we provide is relatively counter-intuitive for the following reason. At first sight, it may appear that a natural strategy for the achievable scheme is to adapt the Han-Kobayashi type message splitting to the $Z$ channel, since the HanKobayashi scheme is optimal in the deterministic $Z$ interference channel ${ }^{1}$. In fact, the achievable strategy described in [13] for the discrete memoryless $Z$ channel does precisely this, by splitting both messages at the second transmitter into private and common messages like the Han-Kobayashi scheme. However, it is not known whether the achieved rate region of [13], which is in terms of several auxilliary variables, represents the entire capacity region for the class of deterministic $Z$ channels considered here. The capacity-optimal achievable scheme we present in this paper, quite surprisingly, does not involve any message splitting and is therefore simpler than the full-fledged Han-Kobayashi scheme. In

\footnotetext{
${ }^{1}$ The $Z$-interference channel is the interference channel where one of the receivers does not face interference from one of the transmitters. The $Z$ channel generalizes the $Z$-interference channel since it has three messages, whereas the latter has only two messages.
} 


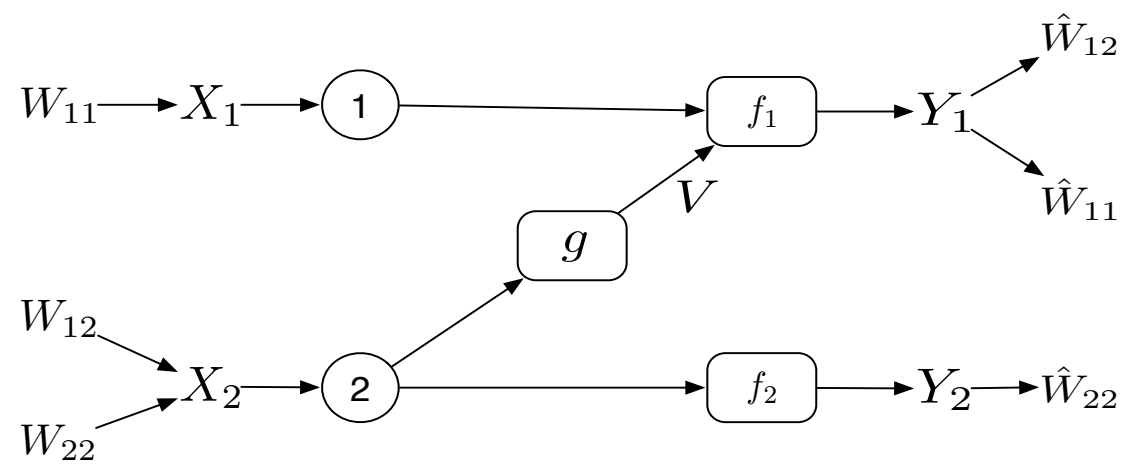

Fig. 1. The Deterministic $Z$ channel

fact, in our achievable scheme, the encoding strategy at the second transmitter mimics the binning strategy that is optimal in the deterministic broadcast channel without a common message [16].

We now proceed to formally define the class of deterministic $Z$ channels considered.

\section{SySTEM MODEL}

The $Z$ channel shown in Figure 1 is characterized by two distributed transmitters (encoders) and two distributed receivers (decoders). Corresponding to the two transmitters, the channel has two discrete inputs $X_{1} \in$ $\mathcal{X}_{1}$ and $X_{2} \in \mathcal{X}_{2}$. Corresponding to the two receivers, there exist two discrete outputs $Y_{1} \in \mathcal{Y}_{1}$ and $Y_{2} \in \mathcal{Y}_{2}$, where

$$
\begin{aligned}
Y_{2} & =f_{2}\left(X_{2}\right) \\
V & =g\left(X_{2}\right) \\
Y_{1} & =f_{1}\left(X_{1}, g\left(X_{2}\right)\right)
\end{aligned}
$$

and $V \in \mathcal{V}$. Note that $f_{1}, f_{2}, g$ are deterministic functions. Similar to the model in [2], we assume that given $X_{1}, Y_{1}$ is invertible, i.e., there exists a function $h$ such that $V=h\left(Y_{1}, X_{1}\right)$. Note that this implies that

$$
H\left(Y_{1} \mid X_{1}\right)=H(V)
$$

There are three independent message sources in the system - one at transmitter 1 , producing message $W_{11} \in$ $\mathcal{W}_{11}$, and two at transmitter 2 , respectively producing messages $W_{12} \in \mathcal{W}_{12}$ and $W_{22} \in \mathcal{W}_{22}$. The sources are assumed to be uniformly distributed over the corresponding message sets. For a code of length $n$, transmitter 1 maps $W_{11}$ to a codeword $\mathbf{X}_{1}^{(n)} \in$ $\mathcal{X}_{1}^{n}$. Transmitter 2 maps message tuple $\left(W_{12}, W_{22}\right)$ to codeword $\mathbf{X}_{2}^{(n)} \in \mathcal{X}_{2}^{n}$. Receiver $j$ intends to decode message(s) $W_{j i},(j, i) \in\{(1,1),(1,2),(2,2)\}$. Correspondingly at receiver $j$, there exist decoding functions $\phi_{j i}^{(n)}: \mathcal{Y}_{j}^{n} \rightarrow \mathcal{W}_{j i}$. The tuple $\left(R_{11}, R_{12}, R_{22}\right)$ is said to be the rate of the code where $R_{j i}=\frac{\log \left|\mathcal{W}_{j i}\right|}{n},(j, i) \in$ $\{(1,1),(1,2),(2,2)\}$. Let

$P_{e}^{(n)}=\sum_{\left\{\begin{array}{c}W_{11}, W_{12} \\ W_{22}\end{array}\right.} \operatorname{Pr}\left(\begin{array}{c}\exists(j, i) \in\{(1,1),(1,2),(2,2)\}, \\ \text { such that } \phi_{j i}\left(\mathbf{Y}_{j}^{(n)}\right) \neq W_{j i} \\ \text { given } W_{11}, W_{12}, W_{22} \text { is encoded }\end{array}\right)$,

where $\mathbf{Y}_{j}^{(n)} \in \mathcal{Y}_{j}^{n}, j=1,2$. The rate-tuple $\left(R_{11}, R_{12}, R_{22}\right)$ is said to be achievable if, for that rate, there exists a sequence of encoding and decoding functions indexed by $n$, such that the error probability $P_{e}^{(n)}$ as defined in (2) vanishes, asymptotically as $n \rightarrow$ $\infty$. The capacity region $\mathcal{C}$ of the $Z$ channel is defined as the set of all achievable rate-tuples. In the remainder of this paper, we follow the notation $\mathbf{X}_{i}^{(n)} \in \mathcal{X}_{i}^{n}, \mathbf{Y}_{i}^{(n)} \in$ $\mathcal{Y}_{i}^{n}, \mathbf{V}^{(n)} \in \mathcal{V}^{n}$.

\section{Main Result : Capacity Region of the DETERMINISTIC $Z$ CHANNEL}

The main result of this paper is stated in the following theorem.

Theorem 3.1: The capacity region of the deterministic $Z$ channel defined in Section II is the set of all ratetuples $\left(R_{11}, R_{12}, R_{22}\right)$ which satisfy, for some product probability distribution $p_{X_{1}}\left(X_{1}\right) p_{X_{2}}\left(X_{2}\right)$, the following.

$$
\begin{aligned}
R_{11} & \leq H\left(Y_{1} \mid V\right) \\
R_{12} & \leq H(V) \\
R_{22} & \leq H\left(Y_{2}\right) \\
R_{12}+R_{11} & \leq H\left(Y_{1}\right) \\
R_{12}+R_{22} & \leq H\left(Y_{2}, V\right) \\
R_{11}+R_{12}+R_{22} & \leq H\left(Y_{1}\right)+H\left(Y_{2} \mid V\right)
\end{aligned}
$$

Before we proceed to prove the theorem, we present the capacity of the $Z$-interference channel.

[2] Corollary 3.2: The capacity region of the $Z$ interference channel, i.e., the $Z$ channel with $W_{12}=\phi$, is the set of all rate-tuples $\left(R_{11}, R_{22}\right)$ which satisfy, for 
some product probability distribution $p_{X_{1}}\left(X_{1}\right) p_{X_{2}}\left(X_{2}\right)$, the following.

$$
\begin{aligned}
R_{11} & \leq H\left(Y_{1} \mid V\right) \\
R_{22} & \leq H\left(Y_{2}\right) \\
R_{11}+R_{22} & \leq H\left(Y_{1}\right)+H\left(Y_{2} \mid V\right)
\end{aligned}
$$

Note that the above result follows from [2]. However, the proof of achievability we provide here is simpler than the full-fledged Han-Kobayashi scheme construction used in [2], since we do not require rate-splitting.

\section{A. Outerbound}

Consider any achievable rate-tuple $\left(R_{11}, R_{12}, R_{22}\right)$. Since receiver 1 is able to decode messages $W_{12}, W_{11}$ from $\mathbf{Y}_{1}^{(n)}$, rates $R_{11}, R_{12}$ must lie in the multiple access channel formed by transmitters and 2, and receiver 1 . This implies that they must satisfy

$$
\begin{aligned}
R_{11} & \leq I\left(Y_{1} ; X_{1} \mid X_{2}\right)=H\left(Y_{1} \mid X_{2}\right) \\
\Rightarrow R_{11} & \leq H\left(Y_{1} \mid V\right) \\
R_{12} & \leq I\left(Y_{1} ; X_{2} \mid X_{1}\right)=H\left(Y_{1} \mid X_{1}\right)=H(V) \\
R_{11}+R_{12} & \leq I\left(Y_{1} ; X_{1}, X_{2}\right)=H\left(Y_{1}\right) .
\end{aligned}
$$

The above equations show that $\left(R_{11}, R_{12}\right)$ must satisfy (3),(4) and (6). The bound (5) on $R_{22}$ is trivial. (7) follows from the cutset bound, with the cut at transmitter 2. Now, using Fano's inequality, we show (8) below. For a codeword of length $n$ and for any $\epsilon>0$, we can write the following.

$$
\begin{aligned}
& n\left(R_{11}+R_{12}+R_{22}-\epsilon\right) \\
& \leq I\left(\mathbf{Y}_{1}^{(n)} ; W_{11}\right)+I\left(\mathbf{Y}_{2}^{(n)} ; W_{22}, W_{12}\right) \\
& =H\left(\mathbf{Y}_{1}^{(n)}\right)-H\left(\mathbf{Y}_{1}^{(n)} \mid W_{11}\right)+I\left(\mathbf{Y}_{2}^{(n)} ; W_{22}, W_{12}\right) \\
& \stackrel{(a)}{\leq} n H\left(Y_{1}\right)-H\left(\mathbf{Y}_{1}^{(n)} \mid W_{11}, \mathbf{X}_{1}^{(n)}\right) \\
& \quad+I\left(\mathbf{Y}_{2}^{(n)}, \mathbf{V}^{(n)} ; W_{22}, W_{12}\right) \\
& \stackrel{(b)}{=} n H\left(Y_{1}\right)-H\left(\mathbf{V}^{(n)}\right)+I\left(\mathbf{Y}_{2}^{(n)}, \mathbf{V}^{(n)} ; W_{22}, W_{12}\right) \\
& \leq n H\left(Y_{1}\right)-H\left(\mathbf{V}^{(n)}\right)+H\left(\mathbf{Y}_{2}^{(n)}, \mathbf{V}^{(n)}\right) \\
& \stackrel{(c)}{\leq} n H\left(Y_{1}\right)+n H\left(Y_{2} \mid V\right) \\
& \quad \Rightarrow R_{11}+R_{12}+R_{22} \leq H\left(Y_{1}\right)+H\left(Y_{2} \mid V\right)
\end{aligned}
$$

The first term in $(a)$ is bounded using the convexity of mutual information, and the fact that conditioning reduces entropy. The second term in $(a)$ is bounded using the fact that conditioning reduces entropy. In $(b)$ we have used (1). In $(c)$ we have again used the fact that conditioning reduces entropy. The final inequality follows from taking $n \rightarrow \infty$. This completes the converse argument. We now proceeed to achievability.

\section{B. Achievable scheme}

Consider a product distribution $p_{X_{1}}\left(X_{1}\right) p_{X_{2}}\left(X_{2}\right)$. We use a random coding argument to show that (3)-(8) is achievable.

1) Encoding Strategy: The first transmitter generates $2^{n R_{11}}$ independent codewords $\mathbf{X}_{1}^{(n)}(m) \in \mathcal{X}_{1}^{n}, m \in$ $\left\{1,2 \ldots, 2^{n R_{11}}\right\}$, generating each element i.i.d according to $p_{X_{1}}\left(X_{1}\right)$. The $i$ th message is encoded using $\mathbf{X}_{1}^{(n)}(i)$.

Note that $p_{X_{2}}\left(X_{2}\right)$ along with the channel induces $p_{X_{2}, V, Y_{2}}\left(X_{2}, V, Y_{2}\right)$ from which marginal distributions $p_{V}(V), p_{Y_{2}}\left(Y_{2}\right)$ and $p_{V, Y_{2}}\left(V, Y_{2}\right)$ can be calculated. Transmitter 2 generates $2^{n A}$ independent sequences $\mathbf{V}^{(n)}(m), m \in\left\{1,2 \ldots 2^{n A}\right\}$, with each symbol drawn i.i.d according to $p_{V}(V)$. These $2^{n A}$ sequences are then distributed uniformly into $2^{n R_{12}}$ bins. The transmitter also generates $2^{n B}$ independent sequences $\mathbf{Y}_{2}^{(n)}(m), m \in\left\{1,2 \ldots 2^{n B}\right\}$ with each symbol drawn i.i.d $p_{Y_{2}}\left(Y_{2}\right)$, and distributes the sequences uniformly into $2^{n R_{22}}$ bins. To encode messages $W_{12}=j, W_{22}=k$, the transmitter chooses a jointly typical $\left(\mathbf{V}^{(n)}, \mathbf{Y}_{2}^{(n)}\right)$ sequence pair in the product bin $(j, k)$, i.e. in $j$ th bin corresponding to the $\mathbf{V}^{(n)}$ sequences, and the $k$ th bin corresponding to the $\mathbf{Y}_{2}^{(n)}$ sequences. If no jointly typical pair is found, then an error is declared. Otherwise, the transmitter encodes the message using the $\mathbf{X}_{2}^{(n)}$ sequence that generates the $\left(\mathbf{V}^{(n)}, \mathbf{Y}_{2}^{(n)}\right)$ sequence picked. The existence of such a $\mathbf{X}_{2}^{(n)}$ sequence is guaranteed, because the channel is deterministic and the pair $\left(\mathbf{V}^{(n)}, \mathbf{Y}_{2}^{(n)}\right)$, by virtue of being jointly typical, has a non-zero probability of occurrence.

Remark: The encoding strategy at transmitter 2 mimics the optimal coding strategy over the deterministic broadcast channel [16].

2) Decoding Strategy: Receiver 1, on receiving $\mathbf{Y}_{1}^{(n)}$, chooses the unique $(i, j)$ such that

$$
\left(\mathbf{V}^{(n)}(j), \mathbf{X}_{1}^{(n)}(i), \mathbf{Y}_{1}^{(n)}\right)
$$

is jointly typical. Then, $W_{11}$ is decoded to be $i$, and $W_{12}$ is decoded using the bin index corresponding to $\mathbf{V}^{(n)}(j)$. If no such pair $(i, j)$ is found, an error is declared. Because of the encoding strategy and the deterministic nature of the channel, receiver 2 receives $\mathbf{Y}_{2}^{(n)}$, whose bin index corresponds to $W_{22}$. If the bin-index of $\mathbf{Y}_{2}^{(n)}$ is unique, then it is decoded using the bin-index. Otherwise an error is declared.

3) Error Analysis : Since the coding scheme is symmetric over all messages, it is sufficient to analyse the probability of error for message $\left(W_{11}, W_{12}, W_{22}\right)=$ $(1,1,1)$. Given that this message tuple was encoded, let $\mathbf{Y}_{1}^{(n)}$ and $\mathbf{Y}_{2}^{(n)}$ respectively represent the sequences 


$$
\begin{aligned}
& E_{1}(i, j)=\left\{\left(\mathbf{Y}_{1}^{(n)}, \mathbf{X}_{1}^{(n)}(i), \mathbf{V}^{(n)}(j)\right) \in \mathcal{A}_{\epsilon}\left(Y_{1}, X_{1}, V\right)\right\} \\
& E_{2}(j, k)=\left\{\begin{array}{c}
\text { At transmitter 2, }\left(\mathbf{V}^{(n)}, \mathbf{Y}_{2}^{(n)}\right) \text { belongs to the } \\
(j, k) \text { th product bin } \Rightarrow\left(\mathbf{V}^{(n)}, \mathbf{Y}_{2}^{(n)}\right) \notin \mathcal{A}_{\epsilon}^{n}\left(V, Y_{2}\right)
\end{array}\right\}
\end{aligned}
$$

received at receivers 1 and 2 . Let $E_{1}, E_{2}$ be sets as defined at the top of this page, where $\mathcal{A}_{\epsilon}^{n}\left(Y_{1}, X_{1}, V\right)$ represents the set of $\epsilon$-jointly typical $\left(\mathbf{Y}_{1}^{(n)}, \mathbf{X}_{1}^{(n)}, \mathbf{V}^{(n)}\right)$ pairs, and $\mathcal{A}_{\epsilon}^{n}\left(V, Y_{2}\right)$ represents the set of $\epsilon$-jointly typical $\left(\mathbf{V}^{(n)}, \mathbf{Y}_{2}^{(n)}\right)$ pairs. Also, let

$$
E_{3}(k)=\left\{\mathbf{Y}_{2}=\mathbf{Y}_{2}^{(n)}(k)\right\} .
$$

Note that $E_{2}(j, k)$ corresponds to the event that no jointly typical $\left(\mathbf{V}^{(n)}, \mathbf{Y}_{2}^{(n)}\right)$ pair is found in the $(j, k)$ th product bin at transmitter 2. Also event $E_{3}(k)$ is equivalent to stating that $\mathbf{Y}_{2}^{(n)}(k)$ and $\mathbf{Y}_{2}^{(n)}$ are jointly typical. The probability of error $P_{e}^{(n)}$, given that $\left(W_{11}, W_{12}, W_{22}\right)=(1,1,1)$, can be bounded as

$$
P_{e}^{(n)}=\operatorname{Pr}\left(E_{2}(1,1)\right)+\operatorname{Pr}\left(\text { Decoding Error } \mid E_{2}^{c}(1,1)\right) .
$$

Consider the set $E_{2}^{c}(1,1)$. This event corresponds to the case that a jointly typical $\left(\mathbf{V}^{(n)}, \mathbf{Y}_{2}^{(n)}\right)$ pair was found in the product bin $(1,1)$. Given that $E_{2}^{c}(1,1)$ occurs, errors can occur at receivers 1 and 2 . Because of the symmetric nature of the coding scheme, the probability of error at receiver 1 is the same no matter which $\mathbf{V}^{(n)}$ sequence chosen by the encoder at transmitter 2. Similarly, the error probability at receiver 2 is the same, no matter which $\mathbf{Y}_{2}^{n}$ is picked at transmitter 2. So, without loss of generality, we can assume that $\left(\mathbf{V}^{(n)}(1), \mathbf{Y}_{2}^{(n)}(1)\right)$ is the jointly typical sequence that was found by the encoder at transmitter 2 in the product bin $(1,1)$. Under this assumption, we can use the union bound to bound the overall error probability as

$$
\begin{aligned}
P_{e}^{(n)} \leq & \operatorname{Pr}\left(E_{2}(1,1)\right)+\operatorname{Pr}\left(E_{1}^{c}(1,1) \mid E_{2}^{c}(1,1)\right) \\
& +\sum_{i=2}^{2^{n R_{11}}} \operatorname{Pr}\left(E_{1}(i, 1) \mid E_{2}^{c}(1,1)\right) \\
& +\sum_{j=2}^{2^{n A}} \operatorname{Pr}\left(E_{1}(1, j) \mid E_{2}^{c}(1,1)\right) \\
& +\sum_{i=2}^{2^{n R_{11}}} \sum_{j=2}^{2^{n A}} \operatorname{Pr}\left(E_{1}(i, j) \mid E_{2}^{c}(1,1)\right) \\
& +\sum_{k=2}^{2^{n B}} \operatorname{Pr}\left(E_{3}(k) \mid E_{2}^{c}(1,1)\right) .
\end{aligned}
$$

It has been shown in [17] that, if

$$
\begin{aligned}
R_{12} & \leq A \\
R_{22} & \leq B \\
R_{12}+R_{22} & \leq A+B-I\left(V ; Y_{2}\right),
\end{aligned}
$$

then $\operatorname{Pr}\left(E_{2}(1,1)\right)$ tends to zero, asymptotically as $n \rightarrow \infty$. Also, by the properties of typical sequences, $\operatorname{Pr}\left(E_{1}^{c}(1,1) \mid E_{2}^{c}(1,1)\right) \rightarrow 0$ as $n \rightarrow \infty$. Now, we bound the probabilities of $E_{1}(1, j), E_{1}(i, 1), E_{1}(i, j)$ and $E_{3}(k)$ given $E_{2}^{c}(1,1)$.

$$
\begin{aligned}
& \operatorname{Pr}\left(E_{1}(1, j) \mid E_{2}^{c}(1,1)\right) \\
& =\sum_{\left(\mathbf{Y}_{1}, \mathbf{X}_{1}, \mathbf{V}\right) \in \mathcal{A}_{\epsilon}^{n}\left(Y_{1}, X_{1}, V\right)} p\left(\mathbf{Y}_{1}^{(n)} \mid \mathbf{X}_{1}^{(n)}\right) p\left(\mathbf{V}^{(n)}\right) p\left(\mathbf{X}_{1}^{(n)}\right) \\
& \leq\left|\mathcal{A}_{\epsilon}^{(n)}\left(Y_{1}, X_{1}, V\right)\right| 2^{-n H\left(Y_{1} \mid X_{1}\right)-n \epsilon} 2^{-n H(V)-n \epsilon} 2^{-n H\left(X_{1}\right)+n \epsilon}
\end{aligned}
$$

$\leq 2^{\left.-n I\left(V ; Y_{1} \mid X_{1}\right)+4 n \epsilon\right)}=2^{-n H\left(Y_{1} \mid X_{1}\right)+H\left(Y_{1} \mid X_{1}, V\right)+4 n \epsilon}$

$=2^{-n H(V)+4 n \epsilon}$

Similarly

$$
\begin{aligned}
\operatorname{Pr}\left(E_{1}(i, 1) \mid E_{2}^{c}(1,1)\right) & \leq 2^{-n I\left(X_{1} ; Y_{1} \mid V\right)+4 n \epsilon} \\
& =2^{-n H\left(Y_{1} \mid V\right)+4 n \epsilon} \\
\operatorname{Pr}\left(E_{1}(i, j) \mid E_{2}^{c}(1,1)\right) & \leq 2^{-n I\left(X_{1}, V ; Y_{1}\right)+4 n \epsilon} \\
& =2^{-n H\left(Y_{1}\right)+4 n \epsilon} \\
\operatorname{Pr}\left(E_{3}(k) \mid E_{2}^{c}(1,1)\right) & =\operatorname{Pr}\left(\mathbf{Y}_{2}^{(n)}=\mathbf{Y}_{2}^{(n)}(k)\right) \\
& \leq 2^{-n H\left(Y_{2}\right)+3 n \epsilon}
\end{aligned}
$$

Using (17)-(20) in (12), we get

$$
\begin{aligned}
P_{e}^{(n)} \leq & \operatorname{Pr}\left(E_{2}(1,1)\right)+\operatorname{Pr}\left(E_{1}^{c}(1,1) \mid E_{2}^{c}(1,1)\right) \\
& \left.+2^{n\left(R_{11}-H\left(Y_{1} \mid V\right)+4 \epsilon\right.}\right)+2^{n(A-H(V)+4 \epsilon)} \\
& +2^{n\left(R_{11}+A-H\left(Y_{1}\right)+4 \epsilon\right)}+2^{n\left(B-H\left(Y_{2}\right)+3 \epsilon\right)}
\end{aligned}
$$

Combining (13)-(15) with the above, we can say that the coding scheme, achieves an average error probability that vanishes as $n \rightarrow \infty$, if the following conditions are staisfied.

$$
\begin{aligned}
R_{11} & \leq H\left(Y_{1} \mid V\right) \\
R_{12} & \leq A \\
R_{11}+A & \leq H\left(Y_{1}\right) \\
R_{22} & \leq B
\end{aligned}
$$




$$
\begin{aligned}
A & \leq H(V) \\
B & \leq H\left(Y_{2}\right) \\
R_{12}+R_{22} & \leq A+B-I\left(V ; Y_{2}\right)
\end{aligned}
$$

We now choose

$$
\begin{aligned}
& A=\min \left(H(V), H\left(Y_{1}\right)-R_{11}\right) \\
& B=H\left(Y_{2}\right)
\end{aligned}
$$

It can be verified that $(A, B)$ satisfy the constraints (23),(25) and (26). Further, on using (28),(29) in (21)(27), the achievable region turns out to be identical to the one described by (3)-(8). This completes the proof of the theorem.

\section{Discussion}

Consider the $Z$-interference channel, i.e., the $Z$ channel with $W_{12}=\phi$. For this channel, interestingly, the scheme does not involve a common message or any other form of message splitting. Therefore, the fullfledged Han-Kobayashi construction is not required for optimality. Further, notice that if

$$
R_{11}>H\left(Y_{1}\right)-H(V)=I\left(X_{1} ; Y_{1}\right)
$$

then $A<H(V)$. In this case, the encoding scheme at transmitter 2 restricts the number of possible sequences of $\mathbf{V}$ to $2^{n A}<2^{n H(V)}$. Therefore, intuitively, the value of $A$ serves to limit the interference caused by transmitter 2 at receiver 1 . Further, note that receiver 1 decodes the $\mathbf{X}_{1}^{(n)}$ sequence that is jointly typical with $\mathbf{Y}_{1}^{(n)}$ and $\mathbf{V}^{(n)}$. This implies that receiver 1 decodes $\mathbf{V}^{(n)}$, which can be interpreted as the variable that implicitly codes the common information from transmitter 2. An interesting open question is whether explicit rate-splitting can be avoided without sacrificing optimality over the (fully connected) deterministic interference channel considered in [2].

On a different note, the deterministic channel we consider captures the deterministic structure introduced in [5] and used in [4], [6], [8] to approximate the capacity of wireless relay, two-user interference, many-to-one and one-to-many networks. Specifically, a deterministic $Z$ channel constructed along the lines of [4] is a special case of the class of channels considered here. Thus, our main result can be used to find the capacity region of this specific deterministic $Z$ channel along the lines of Lemma 4 in [4]. Whether this solution leads to an approximation of the capacity region of the Gaussian $Z$ channel is an interesting area of future work.

\section{CONCLUSION}

We have characterized the capacity region of a class of deterministic $Z$ channels. Interestingly, for the class of $Z$ channels considered, rate-splitting is not required for the optimal achievable scheme. This work, therefore, provides an alternative to the full-fledged Han-Kobayashi construction for the $Z$ interference channel. A natural follow-up question to this work is whether the capacity characterization provided in this paper leads to an approximation of the capacity of the Gaussian $Z$ channel.

\section{REFERENCES}

[1] T. Han, "The capacity region for the deterministic broadcast channel with a common message (corresp.)," Information Theory, IEEE Transactions on, vol. 27, pp. 122-125, Jan 1981.

[2] A. E. Gamal and M. Costa, "The capacity region of a class of deterministic interference channels," IEEE Trans. Inform. Theory, vol. 2, pp. 343-346, March 1982.

[3] R. Etkin, D. Tse, and H. Wang, "Gaussian interference channel capacity to within one bit," arxiv.org, Feb 2007. eprint cs/0702045.

[4] G. Bresler and D. Tse, "The two-user Gaussian interference channel: a deterministic view," European Transactions in Telecoтmunications, vol. 19, no. 4, 2008.

[5] A. S. Avestimehr, S. N. Diggavi, and D. N. C. Tse, "A deterministic approach to wireless relay networks," Arxiv preprint, vol. abs/0710.3777, 2007. http://arxiv.org/abs/0710.3777.

[6] A. S. Avestimehr, S. N. Diggavi, and D. N. C. Tse, "Approximate capacity of Gaussian relay networks," arxiv.org, 2008. eprint $\operatorname{arXiv/0802.3535.~}$

[7] A. S. Avestimehr, A. Sezgin, and D. N. C. Tse, "Approximate capacity of the two-way relay channel: A deterministic approach," Allerton Conference on Communication, Control and Computing, Sep 2008.

[8] G. Bresler, A. Parekh, and D. Tse, "Approximate capacity of the many-to-one interference channel," Sep. 2007. Allerton Conference.

[9] D. Tse and R. Yates, "Fading broadcast channels with state information at the receivers," Allerton Conference on Computing and Control, Sep 2008.

[10] V. R. Cadambe, S. A. Jafar, and S. Shamai, "Interference alignment on the deterministic channel and application to fully connected gaussian interference networks," Information Theory, IEEE Transactions on, vol. 55, pp. 269-274, Jan. 2009.

[11] S. A. Jafar and S. Vishwanath, "Generalized degrees of freedom of the symmetric Gaussian $\mathrm{k}$ user interference channel," Arxiv preprint, vol. abs/0804.4489, 2008. http://arxiv.org/abs/0804.4489.

[12] S. Vishwanath, N. Jindal, and A. Goldsmith, "The "Z" channel," Global Telecommunications Conference, 2003. GLOBECOM '03. IEEE, vol. 3, pp. 1726-1730 vol.3, Dec. 2003.

[13] H.-F. Chong, M. Motani, and H. Garg, "Capacity theorems for the 'Z' channel," Information Theory, IEEE Transactions on, vol. 53, pp. 1348-1365, April 2007.

[14] N. Liu and S. Ulukus, "On the capacity region of the Gaussian Z-channel," Global Telecommunications Conference, 2004. GLOBECOM '04. IEEE, vol. 1, pp. 415-419 Vol.1, Nov.-3 Dec. 2004.

[15] C. Huang, V. R. Cadambe, and S. A. Jafar, "On the capacity and generalized degrees of freedom of the x channel," Arxiv preprint, vol. abs/0810.4741, 2008. http://arxiv.org/abs/0810.4741.

[16] T. Cover, "Comments on broadcast channels," Information Theory, IEEE Transactions on, vol. 44, pp. 2524-2530, Oct 1998.

[17] A. El Gamal and E. van der Meulen, "A proof of Marton's coding theorem for the discrete memoryless broadcast channel (corresp.)," Information Theory, IEEE Transactions on, vol. 27, pp. 120-122, Jan 1981. 